\title{
El farmacéutico, garante de la salud del paciente en la dispensación de ácido acetilsalicílico 500 mg
}

\author{
María Luisa Alonso Núñez, ${ }^{1}$ Amparo Pérez Benajas, ${ }^{2}$ José Calle, ${ }^{3}$ Teresa Eyaralar, ${ }^{4}$ \\ 1. Doctora en Farmacia. Farmacéutica comunitaria en Astorga (León). 2. Doctora en Farmacia. Farmacéutica comunitaria en Vila-Real (Castellón). \\ 3. Farmacéutico comunitario en Toledo. 4. Doctora en Farmacia. Farmacéutica comunitaria. Carbayin Alto (Asturias)
}

\section{PALABRAS CLAVE}

aspirina, farmacias comunitarias, intervención farmacéutica, medicamentos sin prescripción, efectos colaterales, reacciones adversas relacionados con medicamentos

\section{ABREVIATURAS}

AAS: ácido acetilsalicílico. AINE: antiinflamatorio no esteroideo.

DE: desviación estándar. FEDRA: Farmacovigilancia Española. Datos de

Reacciones Adversas.

FT: ficha técnica.

IF: intervención

farmacéutica.

ISRS: inhibidores selectivos de la recaptación de serotonina.

MSP: medicamento susceptible de publicidad. RAM: reacción adversa a medicamento.

\section{KEYWORDS}

aspirin, communities pharmacies, pharmaceutical intervention, nonprescription drugs, drug-related side effects, adverse drug reactions

\section{RESUMEN}

Introducción: La dispensación de medicamentos en España es responsabilidad del farmacéutico. En el caso de los medicamentos no sujetos a prescripción médica (MSP) es el farmacéutico el que asegura su buen uso y puede evitar la aparición de RAM. Uno de los MSP más conocidos es la Aspirina ${ }^{\circledR}, 500 \mathrm{mg}$ de ácido acetilsalicílico (AAS). Este estudio pretende analizar las intervenciones farmacéuticas que se llevan a cabo al dispensar MSP con $500 \mathrm{mg}$ de AAS.

Material y métodos: 18 farmacias comunitarias participaron en este estudio observacional prospectivo. Desde junio de 2013 a junio de 2014 se documentaron todas las intervenciones realizadas a los pacientes que demandaron MSP con $500 \mathrm{mg}$ de AAS y que estuvieran en alguna de las situaciones de riesgo para el consumo de estos medicamentos.

Resultados: De las 3.355 demandas de MSP con $500 \mathrm{mg}$ de AAS contabilizadas, los farmacéuticos intervinieron en 182 ocasiones (el 5,4\% de las veces). Un 20\% de las intervenciones estaban relacionadas con el tiempo de duración del tratamiento, un $24 \%$ con patologías en las que el uso de AAS está contraindicado, otro 24\% con la alteración de pruebas analíticas y un $26 \%$ con la interacción con otros medicamentos.

Discusión: El 5,4\% de las veces que se demandó un MSP con 500 mg de AAS, la intervención del farmacéutico pudo evitar una RAM. Esto hace pensar si es apropiado el plantear que el ASS salga de la farmacia comunitaria. Planteamiento corroborado por el menor número de comunicaciones de RAM relacionadas con AAS de España en relación con el resto de Europa.

The pharmacist as guarantor of patient health in the dispensation of acetylsalicylic acid $500 \mathrm{mg}$

\section{ABSTRACT}

Introduction: Pharmacists hold the responsibility for dispensing of medicines in Spain. When the medicine is an Over the Counter (OTC) one, the pharmacist ensures its good use and prevents the appearance of an ADR (adverse drug reaction). Aspirin ${ }^{\circledR}$ (500 mg of ASA) is one of the best known OTCs. This study analyses the pharmacist's interventions when $500 \mathrm{mg}$ ASA OTCs are dispensed.

Material and methods: 18 community pharmacies took part in this prospective observational study. All interventions made by the pharmacists when patients, who demanded $500 \mathrm{mg}$ ASA OTCs, where in a risky situation for its use, were documented from June 2013 to June 2014.

Results: Pharmacists had to intervene 182 times out of the 3.355 ones a patient demanded a $500 \mathrm{mg}$ ASA OTC (5.4\% of the times). 20\% of interventions where related to the length of treatment, $24 \%$ to pathologies where ASA was contraindicated, other 24\% to lab tests alteration and 26\% to drug-drug interactions.

Discussion: Pharmacist intervention prevented an ADR for the patient 5.4\% of the times a $500 \mathrm{mg}$ ASA OTC was demanded at the pharmacy. This leads us to think carefully about the possibility of taking ASA out of the pharmacy. This fact is supported by the lower number of ADR notifications in Spain compared to other European countries.

Los resultados de la fase piloto fueron presentados en formato póster en el VI Congreso de SEFAC de Málaga, mayo 2014.

Financiación: Ninguna ajena.

Conflicto de intereses: Los autores declaran no existir conflicto de intereses en relación con el contenido del artículo.

Cite este artículo como: Alonso ML, Pérez A, Calle J, Eyaralar T. El farmacéutico, garante de la salud del paciente en la dispensación de ácido acetilsalicilico 500 mg. Farmacéuticos Comunitarios. 2014 Dec 01; 6(4):5-13. doi:10.5672/FC.2173-9218.(2014/Vol6).004.02

Autora para correspondencia: Maria Luisa Alonso Núñez (lualnu@yahoo.es).

ISSN 1885-8619 @SEFAC (Sociedad Española de Farmacia Familiar y Comunitaria). Todos los derechos reservados. 


\section{Introducción}

La dispensación de medicamentos en España es responsabilidad del farmacéutico, independientemente del entorno en que se realice (farmacias comunitarias o servicios de farmacia hospitalarios) (1), entendiéndose como dispensación el servicio profesional del farmacéutico encaminado a garantizar, tras una evaluación individual, que los pacientes reciban $\mathrm{y}$ utilicen los medicamentos de forma adecuada a sus necesidades clínicas, en las dosis precisas según sus requerimientos individuales, durante el periodo de tiempo adecuado, con la información para su correcto uso, y de acuerdo con la normativa vigente (2). Cuando a la entrega de medicamentos legalmente realizada se le añade una evaluación de su adecuación al paciente en el momento de la dispensación, también se le añade una mayor responsabilidad del farmacéutico a la actuación. Así se cumple la exigencia básica de cualquier servicio de atención farmacéutica, consistente en adquirir una parte de responsabilidad en los resultados de la farmacoterapia de cada individuo (2).

Las condiciones en que se realiza dicha dispensación dependerán de la clasificación de los medicamentos, y esta clasificación a su vez se establece en función del riesgo que su uso puede suponer para el usuario concreto o para la sociedad. Se clasifican en medicamentos sujetos a prescripción y medicamentos no sujetos a prescripción $(1,3)$. Estos últimos en la base de datos BOT Plus del Consejo General de Colegios Oficiales de Farmacéuticos constan como medicamentos susceptibles de publicidad bajo el acrónimo MSP (4)

La Ley 29/2006, de 26 de julio, de garantías y uso racional de los medicamentos y productos sanitarios indica que la Agencia Española de Medicamentos y Productos sanitarios podrá calificar como medicamentos no sujetos a prescripción médica aquéllos que vayan destinados a procesos o condiciones que no necesiten un diagnóstico preciso y cuyos datos de evaluación toxicológica, clínica o de su utilización y vía de administración no exijan prescripción médica, de modo que dichos medicamentos puedan ser utilizados para autocuidado de la salud, mediante su dispensación en la oficina de farmacia por un farmacéutico, que informará, aconsejará e instruirá sobre su correcta utilización (1).

Uno de esos medicamentos clasificados como MSP es Aspirina ${ }^{\circledR}$, y aunque su uso como analgésico se ha ido desplazando hacia otros antiinflamatorios como ibuprofeno y paracetamol, podemos decir que su uso está ligado tradicionalmente a la automedicación, conservando su hueco en muchos botiquines domésticos.

El ácido acetilsalicílico (principio activo de Aspirina ${ }^{\circledR}$ ), o AAS, para su uso no cardiovascular está sujeto a prescripción médica en cinco presentaciones comerciales $\left(\right.$ AAS $^{\circledR} 500 \mathrm{mg}$ 20 comprimidos, Dolmen ${ }^{\circledR} 10$ comprimidos, Dolmen ${ }^{\circledR} 20$ comprimidos, Dolvirán ${ }^{\circledR} 10$ supositorios y Dolvirán ${ }^{\circledR} 20$ comprimidos). Sin embargo, la mayor parte de los medicamentos que contienen AAS no requieren de dicha prescripción médica y son considerados MSP. Los MSP que contienen 500 mg de AAS (cantidad de AAS presente en la Aspirina ${ }^{\circledR}$ ) son 23 y se dividen en dos grupos terapéuticos: el N02BA, analgésicos y antipiréticos derivados del ácido salicílico; y el grupo R05X, productos combinados para el resfriado (tabla 1 ).

Teniendo en cuenta que se define reacción adversa a un medicamento (RAM) como cualquier respuesta a un medicamento que sea nociva y no intencionada, y que tenga lugar a dosis que se apliquen normalmente en el ser
Tabla 1 Medicamentos susceptibles de publicidad que contienen $500 \mathrm{mg}$ de AAS

\begin{tabular}{|c|l|l|}
\hline Código & Nombre & Grupo terapéutico \\
\hline 8822584 & Acyflox (500/200 mg 20 comprimidos) & N02BA \\
\hline 6603697 & Aspirina (500 mg 10 comprimidos efervescentes) & N02BA \\
\hline 9805739 & Aspirina (500 mg 10 comprimidos masticables) & N02BA \\
\hline 6545713 & Aspirina (500 mg 10 sobres granulado) & N02BA \\
\hline 6614983 & Aspirina (500 mg 2 comprimidos efervescentes) & N02BA \\
\hline 6612200 & Aspirina (500 mg 2 sobres granulado) & N02BA \\
\hline 7127864 & Aspirina (500 mg 20 comprimidos) & N02BA \\
\hline 6603703 & Aspirina (500 mg 20 comprimidos efervescentes) & N02BA \\
\hline 6615232 & Aspirina (500 mg 20 sobres granulado) & N02BA \\
\hline 7095279 & Aspirina Complex (10 sobres) & R05X \\
\hline 7830504 & Aspirina Gervasi (500 mg 20 comprimidos) & N02BA \\
\hline 8329496 & Aspirina Plus (500/50 mg 20 comprimidos) & N02BA \\
\hline 7241201 & Cafiaspirina (500/50 mg 20 comprimidos) & N02BA \\
\hline 8764174 & Calmante Vitaminado Pérez Giménez (10 Comprimidos) & N02BA \\
\hline 8764259 & Calmante Vitaminado Pérez Giménez (20 comprimidos) & N02BA \\
\hline 6815281 & Couldina (20 comprimidos efervescentes ) & R05X \\
\hline 6969489 & Couldina C (10 comprimidos efervescentes) & R05X \\
\hline 7895039 & Couldina C (20 comprimidos efervescentes) & R05X \\
\hline 6893982 & Couldina Instant (10 sobres efervescentes) & R05X \\
\hline 6893807 & Couldina Instant (20 sobres efervescentes) & R05X \\
\hline 9069339 & Okaldol (500/250 mg 20 comprimidos dispersables) & N02BA \\
\hline 8736799 & Okaldol (500/250 mg 4 comprimidos dispersables) & N02BA \\
\hline 9967772 & Okaldol con cafeina (500/250/30 mg 12 comprimidos masticables) & N02BA \\
\hline
\end{tabular}


humano para la profilaxis, el diagnóstico o el tratamiento de enfermedades, o para la restauración, corrección o modificación de funciones fisiológicas (incluyendo también todas las consecuencias clínicas perjudiciales derivadas de la dependencia, abuso y uso incorrecto de medicamentos, incluyendo las causadas por el uso fuera de las condiciones autorizadas y las causadas por errores de medicación) (5), la consideración de MSP no exime de riesgo a estos medicamentos. Por esta razón su venta se regula por ley bajo la responsabilidad del farmacéutico y con las garantías que se exigen a las farmacias comunitarias (1).

Según datos de la Agencia Europea del Medicamento, dentro del espacio económico europeo se notifican al mes 2.005 casos de RAM graves referidas al uso de AAS (6). Esto significa que en el espacio económico europeo 67 personas se ven afectadas cada día por posibles RAM graves debidas al uso de AAS, siendo éstas notificadas por los profesionales sanitarios que los atienden.

En España se dispensan y/o venden al mes una media de 657.972 medicamentos no sujetos a prescripción médica que contienen $500 \mathrm{mg}$ de AAS (datos obtenidos desde octubre de 2013 a septiembre de 2014 y proporcionados por la empresa Health Marketing Research -HMR-), y según los últimos datos extraídos de la base de datos del sistema de farmacovigilancia español FEDRA, en un periodo de dos años (de 2011 a 2013) se recogieron 328 notificaciones de reacciones adversas relacionadas con el AAS distribuyéndose por órganos/sistemas afectados de la siguiente manera: trastornos gastrointestinales (153 casos), trastornos de la sangre y del sistema linfático (77 casos), trastornos de la piel y del tejido subcutáneo (49 casos), trastornos respiratorios, torácicos y mediastínicos (40 casos), trastornos del sistema nervioso (40 casos), trastornos renales y urinarios (30 casos), trastornos generales y alteraciones en el lugar de administración (30 casos), trastornos vasculares (30 casos), trastornos músculo-esqueléticos (13 casos), lesiones traumáticas, intoxicaciones y complicaciones de procedimientos terapéuticos (14 casos), y trastornos cardíacos (10 casos) (7).

Cuando un paciente solicita a un farmacéutico un medicamento que no necesita prescripción médica, una vez explorados signos y síntomas y verifi- cado que la solicitud se adecua a la indicación recogida en la ficha técnica, es responsabilidad del farmacéutico asegurarse de que el paciente no presenta ninguna situación fisiológica, patológica o medicamentosa que haga que el uso del medicamento solicitado precise de una vigilancia especial en el tiempo o simplemente necesite una derivación al médico o el uso de otro medicamento. Se trata por tanto de un acto clínico en el que el farmacéutico procesa la información de que dispone, aplica sus conocimientos farmacológicos y toma la decisión de no dispensar o dispensar con la información que considera adecuada. Este acto profesional, que realizan a diario los farmacéuticos en las farmacias comunitarias, es el que garantiza a la población que su salud está protegida (8), además de ser la razón por la que en España la "venta" de medicamentos está restringida a las farmacias comunitarias, distinguiéndola de otros países europeos donde la venta de algunos medicamentos no requiere la presencia de un farmacéutico (9).

\section{Objetivos}

Ya que una intervención farmacéutica (IF) es una decisión clínica y las decisiones clínicas requieren la presencia de un profesional sanitario los objetivos de este trabajo son:

1. Objetivo general: estudiar, desde el punto de vista sanitario, el impacto del acto profesional del farmacéutico en la dispensación de AAS en pacientes individuales.

2. Objetivo específico: caracterizar las IF por riesgo de uso en personas que solicitan AAS, solo o asociado, en dosis de $500 \mathrm{mg}$ tras la evaluación por el farmacéutico de dicho riesgo en cada paciente concreto.

\section{Material y métodos}

Se trata de un estudio observacional prospectivo multicéntrico con un componente de intervención. Para este trabajo se seleccionaron 18 farmacias comunitarias, inscritas voluntariamente, de las que estaban participando en el momento del inicio del estudio en el programa Comparándonos de la Sociedad Española de Farmacia Familiar y Comunitaria, SEFAC (10), y que se encuentran situadas en diferentes comunidades autónomas y con diferentes características de tamaño y tipo de farmacia (el volumen de ventas/dispensaciones de medicamentos varía desde 3.933 a 50.508 actuaciones al mes). Desde junio de 2013 a junio de 2014 se documentaron y enviaron a SEFAC cuantas intervenciones se fuesen haciendo a pacientes que habian solicitado alguno de los medicamentos con AAS que constan en la tabla 1 y en los que podia existir alguna sospecha de situación de riesgo por la administración del mismo, contraindicaciones o precauciones de uso, que denominamos "situaciones patológicas". Para ello se utilizó la hoja de registro que se muestra en la figura 1. Dicha hoja de registro está basada en la ficha técnica de la Aspirina ${ }^{\circledR}$ por cuestiones de tratamiento informático y por ser el medicamento más usado. En el estudio se han incluido asociaciones de principios activos así que en estos casos, aunque los datos de indicación no coinciden al 100\%, comparten las indicaciones de dolor y fiebre.

Todas las farmacias realizaron una fase previa de pilotaje utilizando la metodología acordada durante 10 días previos al estudio. La segunda fase se realizó tras la puesta en común y aceptación por los participantes de los cambios introducidos y utilizando como protocolo el documento final que se confeccionó ad hoc (http:// www.farmaceuticoscomunitarios.org/ anexos/FC2014_6-4-AAS-ANEXO. pdf) para unificar la asistencia sanitaria prestada y el registro de datos. En la primera fase del estudio se consideró intervención cuando el farmacéutico identificaba una situación de riesgo consignada en la hoja de registro. Además, en la primera fase del estudio se detectó un error en la variable contraindicación embarazo (primer trimestre) y que no se había contemplado registrar edad del usuario, nombre del medicamento solicitado o entregado, ni el grupo terapéutico. En la segunda fase se siguieron las instrucciones según el protocolo aprobado.

La recogida de datos se realizó de forma automática en las farmacias comunitarias que contaban con el programa informático NIXFARMA (13 farmacias) o BITFARMA (2 farmacias) y de forma manual en el resto. Los datos cualitativos se expresarán como porcentajes y los cuantitativos como media con su desviación estándar (DE), indicándose el rango de resultados. 


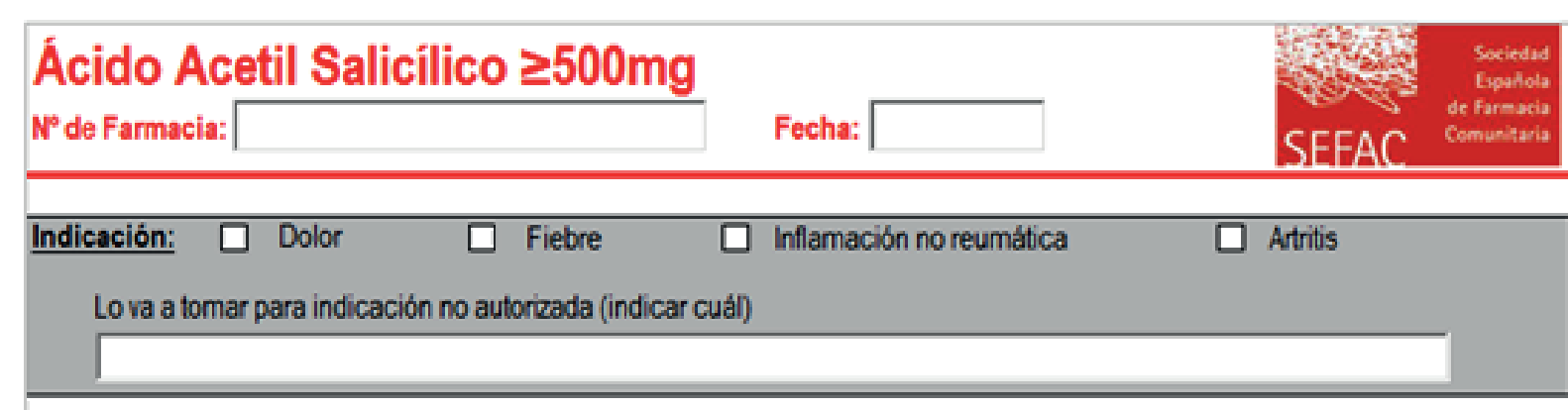

\section{Situaciones fisiológicas de contraindicación}

Niño menor de 16 años

Embarazada en el tercer trimestre

$\square \quad$ Lactancia

$\square \quad$ Más de 3 bebidas alcoholicas al dia

2. Situaciones de tiempo (derivar al médico)

Lleva más de 5 dias tomándolo para el dolor $\quad \square \quad$ Lleva más de 3 dias tomándolo para la fiebre

\section{Situaciones patológicas de contraindicación/precaución}

Alérgico a salicilatos

Ülcera gastroduodenal

Antecedentes de hemorragias

Asma

Insuficiencia renal/hepática

Se va a hacer analitica (indicar cuáles)

$\begin{array}{lll}\square & \text { Bilimubina } & \square \\ \square & \text { Digoxina } & \square \\ \square & \text { Triglicéridos } & \square\end{array}$

Triglicéridos

$\square$
Hemofilia

Hipoprotrombinemia

Gota

Menorragia

Hipertensión arterial

Acaba de extraerse una muela

Transaminasas
Creatinina
Glucosa

Grupo terapéutico

Medicamento

Dosis/dia

AINEs

Corticoides

Diuréticos

Inhibidores serotonina

Anticoagulantes

Antiagregantes

Tromboliticos

Insulinas

Ciclosporina

Vancomicina

Interferon alfa

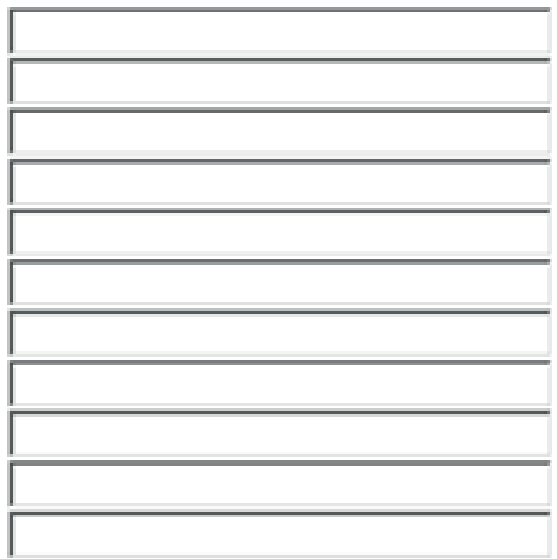

Colesterol

Ácido úrico

T3 y T4

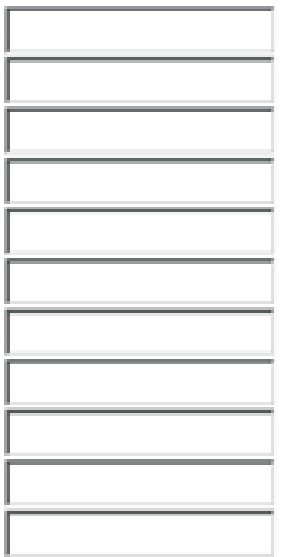

Más adelante evaluaremos en SEFAC lo que habria pasado sin tu actuación en este caso. Para ello, necesitamos saber los siguientes datos: Edad, Dosis de AAS ...

Y seria conveniente que lo tengas identificado internamente porque, al ser el estudio totalmente anónimo, puede que tengamos que ponernos en contacto contigo posteriormente para recabar algủn dato necesario

$$
\mathrm{N}^{\mathrm{p}} \text { paciente }
$$




\section{Resultados}

Durante el periodo de estudio se contabilizaron 3.355 demandas de MSP con $500 \mathrm{mg}$ de AAS con una media de 223,6 (DE= 202,6; Mín=37, Máx=626) por farmacia. En 182 ocasiones el farmacéutico tuvo que intervenir al identificar en el paciente alguna de las precauciones o contraindicaciones contempladas en la ficha técnica de la Aspirina ${ }^{\circledR}$ y recogidas en este estudio. En 182 ocasiones (5,4\%) el farmacéutico tuvo que intervenir al identificar en el paciente alguna de las precauciones o contraindicaciones contempladas en la ficha técnica de Aspirina ${ }^{\circledR}$ y recogidas en este estudio. La media de intervenciones por farmacia en el periodo de estudio es de 10,1 (DE= 13,7; Mín=1, Máx=60).

La tabla 2 muestra las distintas situaciones de riesgo que promovieron la intervención del farmacéutico y el número absoluto de intervenciones que se realizaron en cada caso y en cada farmacia. Se desglosan los datos de mayor relevancia como son la situación fisiológica, un uso previo durante un tiempo superior al que se considera de evolución normal del proceso para automedicación, las precauciones de uso y las interacciones.

En la figura 2 está representada la distribución de las intervenciones según el tipo de problema detectado. Prácticamente todos los problemas detectados están relacionados a partes iguales con el tiempo de duración del tratamiento, con patologías en las que está contraindicado el uso de AAS, con alteración de pruebas analíticas y con la interacción con otros medicamentos.

\section{Discusión}

Los resultados de este estudio nos confirman la importancia de la presencia de un farmacéutico en la dispensación de medicamentos solicitados para automedicación debido al riesgo que supondría la falta de información o los errores de interpretación del contenido del prospecto por parte de la persona que lo va a utilizar. Aunque estos medicamentos se podrían considerar seguros por no estar sometidos a prescripción médica, el riesgo cero no existe y por otro lado, la autonomía del paciente no exime al farmacéutico de su responsabilidad como garante sanitario de que ese medicamento va a ser utilizado en las condiciones legalmente aceptadas y que son las recogidas en la ficha técnica y en el prospecto (1).

En los resultados se observa que la demanda es de 3.355 MSP con $500 \mathrm{mg}$ de AAS y que la amplitud del intervalo de dicha demanda por farmacia posiblemente esté relacionada con el diferente número de ventas/ dispensaciones de las farmacias participantes (10). Esto podría explicar también las diferencias en el número de intervenciones por farmacia.

En cuanto a patologías contraindicadas cabe destacar la hipertensión arterial por su alta aparición en los datos (55\% de los casos de patologías) y el asma (16\% de los casos de patologías) por la gravedad de las RAM que se pueden producir $(11,12)$. En el caso de interacciones farmacológicas las más comunes son las que ocurren con otros medicamentos antiinflamatorios no esteroideos (AINE) $(13,14)$ (41\% de los casos de interacciones), seguidas por las producidas con los diuréticos (28\% de los casos), de menor proporción pero de mayor importancia por la gravedad de las RAM que producen $(15,16)$.

\section{Embarazo}

No se encontró ningún caso de embarazo en tercer trimestre pero si el farmacéutico hubiera decidido no dispensar AAS en un caso así, el medicamento indicado como alternativa terapéutica para dolor y fiebre es el paracetamol $(17,18)$.

\section{Niños menores de 16 años}

El sindrome de REYE es una enfermedad inflamatoria cerebral y hepática que se asoció a la utilización de Aspirina ${ }^{\circledR}$ en niños con varicela y gripe, dando lugar a la retirada del mercado en el año 2003 de Aspirina infantil ${ }^{\circledR}$ y a la contraindicación de uso para menores de 16 años de cualquier preparado que contuviese AAS (19). Actualmente su uso es residual, ocupando su lugar ibuprofeno y paracetamol. Sin embargo, en este estudio queda patente el hecho de que todavía hay quien desconoce ese riesgo y el motivo de la contraindicación.

\section{Tiempo de uso previo}

El tiempo de evolución de un proceso se considera como un criterio para definir la automedicación y como consecuencia es una alerta para que el paciente sea derivado a otro nivel de la asistencia sanitaria. En el trabajo se encontraron 37 pacientes $(20 \%$ del total) que solicitaban el medicamento para continuar su tratamiento, bien para el dolor (33 casos) o bien para la fiebre (4 casos), durante un tiempo superior al considerado seguro y que a priori deberían ser derivados para evitar enmascarar un proceso que requiere atención médica.

\section{Interferencias analíticas}

La disparidad en los resultados obtenidos en la variable interferencia con pruebas analíticas nos hace pensar en diferencias importantes entre los farmacéuticos participantes a la hora de recoger e interpretar estos datos. Acceder a este tipo de información es un hecho que limita la actuación del farmacéutico y es motivo constante de reivindicación por parte del colectivo. La interferencia de los medicamentos con pruebas analíticas puede dar lugar a diagnósticos desacertados, siendo las más relevantes aquellas que pueden indicar alteración hepática o daño renal, y las determinaciones de las hormonas T3 y T4 que son especialmente importantes por la dificultad que entraña el ajuste de dosis de hormonas tiroideas.

\section{Situaciones de enfermedad} concomitante e interacciones con otros medicamentos

En la discusión se decidió agrupar interacciones y enfermedades puesto que el riesgo de las interacciones cobra mayor relevancia dependiendo de las enfermedades del paciente. Por ejemplo, un paciente con antecedentes de úlcera tendrá un riesgo incrementado si utiliza un corticoide o un inhibidor selectivo de la recaptación de la serotonina (ISRS) y para automedicación solicita AAS, que otro paciente que no tenga antecedentes de úlcera. De la tabla de resultados (tabla 2) no se puede concluir ninguna clasificación de pacientes que presenten más de una situación de riesgo.

Se realizan un total de 44 intervenciones en el apartado de situaciones patológicas, que suponen el 24\% del total. La dificultad del farmacéutico ante la falta de información objetiva es discernir si se trata de una contraindicación o de una precaución. 


\begin{tabular}{|c|c|c|c|c|c|c|c|c|c|c|c|c|c|c|c|c|c|c|c|c|}
\hline 1 & Indic. no autorizada & & & & 1 & 2 & 1 & & & & & & 2 & & & & & & & 6 \\
\hline A & Indicación & 0 & 0 & 0 & 1 & 2 & 1 & 0 & 0 & 0 & 0 & 0 & 2 & 0 & 0 & 0 & 0 & 0 & 0 & 6 \\
\hline 2 & Menor 16 años & & & & & & & & & & & & & & 1 & & & & & 1 \\
\hline 3 & Embarazo $3^{\circ}$ trimestre & & & & & & & & & & & & & & & & & & & 0 \\
\hline 4 & Lactancia & & & & & & & & & & & & & & & & & & & 0 \\
\hline 5 & Más de 3 bebidas día & & & & & & & & & & & & 3 & & & & 1 & & & 4 \\
\hline B & Fisiológicas & 0 & 0 & 0 & 0 & 0 & 0 & 0 & 0 & 0 & 0 & 0 & 3 & 0 & 1 & 0 & 1 & 0 & 0 & 5 \\
\hline 6 & Más de 5 dias dolor & 2 & 1 & 1 & 9 & 1 & & 5 & 1 & & & 2 & 1 & & 4 & & 1 & 2 & 3 & 33 \\
\hline 7 & Más de 3 dias fiebre & & & 1 & & & & & 1 & & & & 2 & & & & & & & 4 \\
\hline C & Tiempo & 2 & 1 & 2 & 9 & 1 & 0 & 5 & 2 & 0 & 0 & 2 & 3 & 0 & 4 & 0 & 1 & 2 & 3 & 37 \\
\hline 8 & Alergia salicilatos & & & & & & & & & & & & & & & & & & 1 & 1 \\
\hline 9 & Úlcera gastroduodenal & 1 & 1 & & & & & 2 & & & & & 1 & & & & & & & 5 \\
\hline 10 & Anteced. hemorragia & & & & & & & 1 & & & & & & & & & & & & 1 \\
\hline 11 & Asma & & & 1 & 1 & & & & & & & & & & 1 & 1 & & 1 & 2 & 7 \\
\hline 12 & Insuf. renal/hepática & & & & 2 & 1 & & & & & & & & & & & & & 1 & 4 \\
\hline 13 & Hemofilia & & & & & & & & & & & & & & & & & & & 0 \\
\hline 14 & Hemoprotombinemia & & & & & & & & & & & & & & & & & & & 0 \\
\hline 15 & Gota & & & & & & & 1 & & & & & & & & & & & & 1 \\
\hline 16 & Menorragia & & & & & & & & & & & & 1 & & & & & & & 1 \\
\hline 17 & Hipertension arterial & 1 & 1 & 1 & 8 & & 4 & 1 & & & 1 & & 1 & 1 & 1 & 1 & & & 3 & 24 \\
\hline 18 & Se extrajo muela & & & & & & & & & & & & & & & & & & & 0 \\
\hline D & Patológicas & 2 & 2 & 2 & 11 & 1 & 4 & 5 & 0 & 0 & 1 & 0 & 3 & 1 & 2 & 2 & 0 & 1 & 7 & 44 \\
\hline 19 & An. transaminasas & & & 1 & 3 & & & & & & & & & & & & & & & 4 \\
\hline 20 & An. creatinina & & & & 2 & & & & & & & & & & & & & & & 2 \\
\hline 21 & An. glucosa & & & & 7 & & & 1 & & & & 1 & & & & & & & 1 & 10 \\
\hline 22 & An. bilirrubina & & & & & & & & & & & & & & & & & & & 0 \\
\hline 23 & An. digoxina & & & & & & & & & & & & & & & & & & & 0 \\
\hline 24 & An. triglicéridos & & & 1 & 6 & & & & & & & & & & & & & & 1 & 8 \\
\hline 25 & An. colesterol & & & 1 & 6 & & & 2 & & & & 1 & & & & & & & 3 & 13 \\
\hline 26 & An. ácido úrico & & & 1 & 5 & & & & & & & & & & & & & & & 6 \\
\hline 27 & An. T3, T4 & & & & & & & & & & & & & & & & & & & 0 \\
\hline$E$ & Analiticas & 0 & 0 & 4 & 29 & 0 & 0 & 3 & 0 & 0 & 0 & 2 & 0 & 0 & 0 & 0 & 0 & 0 & 5 & 43 \\
\hline 28 & AINES & 2 & 1 & & 1 & & 3 & 3 & & 2 & & & & 1 & 2 & & & 1 & 3 & 19 \\
\hline 29 & Corticoides & & & & 1 & & & & & & & & 1 & & & & 1 & & 2 & 5 \\
\hline 30 & Diuréticos & & & & 5 & 1 & 1 & & & & & 1 & & & 2 & 1 & 1 & & 1 & 13 \\
\hline 31 & Inh. serotonina & & & & & & & & & & & & & & 1 & & & & 1 & 2 \\
\hline 32 & Anticoagulantes & & & & 2 & & & & & & & & & & & & & & 1 & 3 \\
\hline 33 & Antiagregantes & & & & & 1 & & & & & & & & & & & & & & 1 \\
\hline 34 & Trombolíticos & & & & & & & & & & & 1 & & & & & & & & 1 \\
\hline 35 & Insulinas & & & & 1 & & & & & & & 1 & & & & & & & 1 & 3 \\
\hline 36 & Ciclosporina & & & & & & & & & & & & & & & & & & & 0 \\
\hline 37 & Vancomicina & & & & & & & & & & & & & & & & & & & 0 \\
\hline 38 & Interferón alfa & & & & & & & & & & & & & & & & & & & 0 \\
\hline G & Interacciones & 2 & 1 & 0 & 10 & 2 & 4 & 3 & 0 & 2 & 0 & 3 & 1 & 1 & 5 & 1 & 2 & 1 & 9 & 47 \\
\hline $\mathrm{H}$ & Total intervenciones & 6 & 4 & 8 & 60 & 6 & 9 & 16 & 2 & 2 & 1 & 7 & 12 & 2 & 12 & 3 & 4 & 4 & 24 & 182 \\
\hline
\end{tabular}

Tabla 2 Número de intervenciones realizadas por farmacia y tipo de problema 


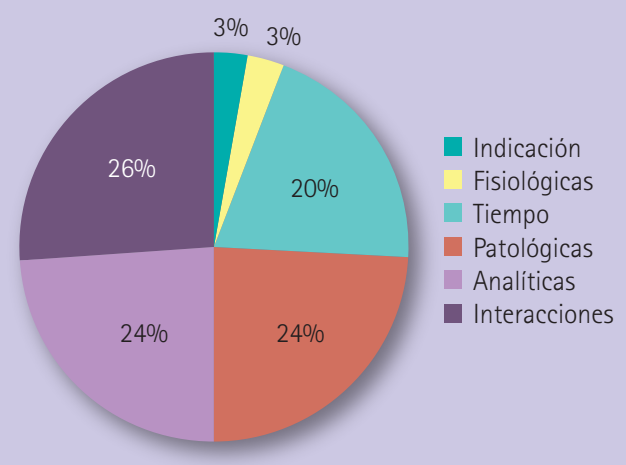

Tiempo

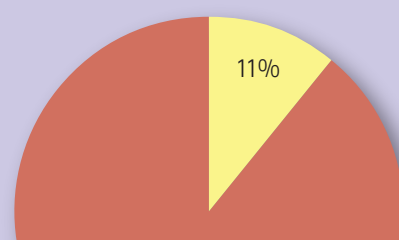

$89 \%$

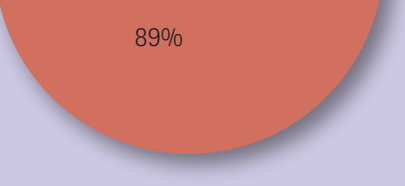

Más de 5 días dolor Más de 3 dias fiebre

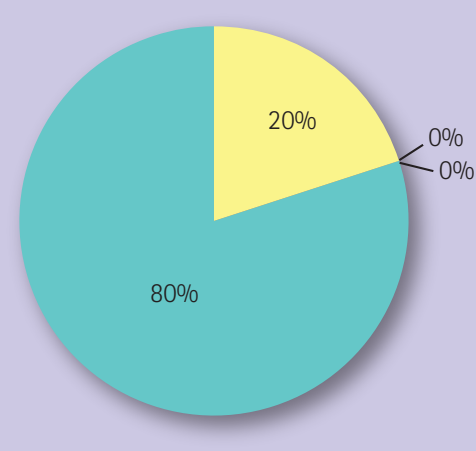

Patológicas

$0 \% \quad 2 \%$

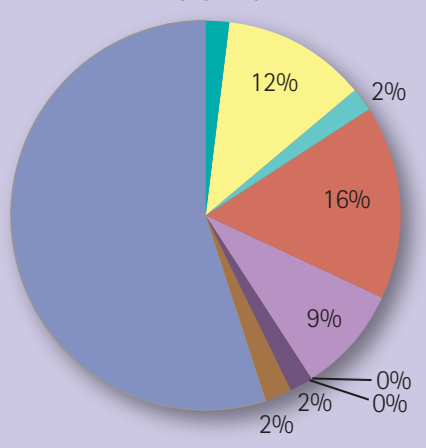

- Alergia Salicilatos

Úlcera gastroduodenal

Antecedentes de hemorragia

- Asma

- Insuficiencia renal/hepática

Hemofilia

- Hemoprotombinemia

- Gota

- Menorragia

- Hipertensión arterial

- Se extrajo muela

\section{Analíticas}

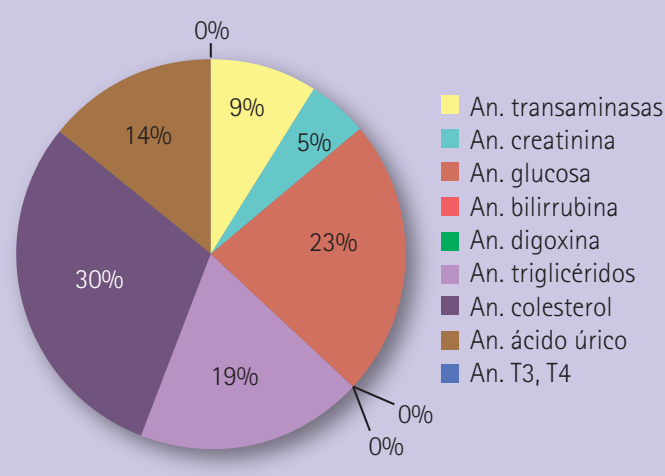

Interacciones

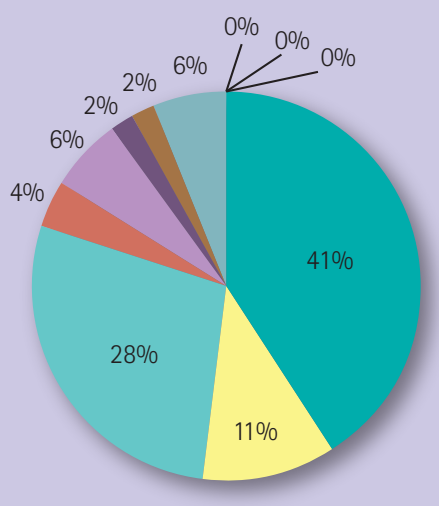

AINES

Corticoides

Diuréticos

Inh. Serotonina

Anticoagulantes

Antiagregantes

Trombilíticos

Insulinas

Ciclosporina

Vancomicina

Interferón alfa 
Esto que parece poco relevante tiene una gran importancia ya que ante una contraindicación se debe bloquear la dispensación y tomar la decisión clínica de proponer una alternativa terapéutica más segura o derivar al médico. Por el contrario, en una precaución de uso el acto profesional consiste en evaluar el balance riesgo-beneficio y decidir si dispensar o no. Este problema se presentó en las dispensaciones a los 7 pacientes con asma, a los 5 con úlcera y a los 4 con insuficiencia renal y hepática.

El dato de función renal es clave para el farmacéutico a la hora de decidir qué medicamentos y a qué dosis puede dispensar por si fuera necesario bloquear una dispensación o proponer un ajuste de dosis. Esto adquiere especial relevancia en personas mayores en las que el deterioro renal es un hecho fisiológico, y que además pueden estar utilizando medicamentos para la hipertensión arterial o para la insuficiencia cardíaca. Además, estas enfermedades son tratadas con medicamentos que dañan el riñón o que interaccionan con el AAS incrementando el riesgo de fallo renal, bien por disminuir la eliminación del medicamento o bien porque producen daño renal propiamente dicho. Hablamos de la asociación de AINE con diuréticos, inhibidores de la enzima convertidora de angiotensina (IECA) y antagonistas de los receptores de angiotensina II (ARA II). El mecanismo de la interacción entre AAS y diuréticos es bien conocido y los datos de manifestación de RAM asociados a su uso conjunto son relevantes y abundantes $(15,16)$.

En cuanto a la función hepática, para el farmacéutico es mucho más difícil disponer del dato del grado de insuficiencia, que vendría dado, entre otros, por el test de Child-Pugh (20).

El riesgo de hemorragia probablemente sea la RAM más frecuente y mejor documentada a través de los casos recogidos en la bibliografía (2124). En este estudio 5 pacientes refirieron tener antecedentes de úlcera y la utilización de medicamentos cuyo uso conjunto incrementa el riesgo de hemorragias, bien por daño directo o bien por afectar a la coagulación, como es el caso de los corticoides (5 casos), los inhibidores selectivos de la recaptación de serotonina (ISRS) (2 casos), los anticoagulantes (3 casos) o los trombolíticos (1 caso), además de los 19 que utilizaban otro AINE.

En el estudio se pretendía registrar el nombre del medicamento identificado en la interacción, pero no se dispone de este dato probablemente por la dificultad que tienen los pacientes para identificar los medicamentos por nombre comercial. La estrategia para abordar este problema fue hacer una aproximación intentando obtener del paciente la información de para qué usa medicamentos.

Lo que más sorprende de este apartado es el dato de los tres pacientes que utilizaban un anticoagulante, ya que suelen estar bien informados del riesgo de la automedicación, especialmente con AINE y en la información que se les suministra cuando inician el tratamiento, por ejemplo con Sintrom ${ }^{\circledR}$, se les advierte de lo que pueden tomar o no en caso de dolor y fiebre, así como de advertir en cada momento a los profesionales sanitarios de que utilizan ese medicamento.

\section{Limitaciones}

La selección de farmacias no fue aleatoria sino que se realizó entre las que voluntariamente se apuntaron de entre las que participaban en un estudio paralelo (10). Constituye igualmente una limitación el no disponer de las características de las farmacias en cuanto al tipo de población atendida, ya que las farmacias consideradas de paso, a priori, tienen más demandas para automedicación que las que atienden a población con fácil acceso al sistema sanitario como puede ser la farmacia rural o de barrio.

Por otra parte, la clase social y el nivel educativo de los pacientes son condiciones a tener en cuenta en los estudios sobre los riesgos y beneficios de la automedicación ya que la pobreza y el nivel cultural bajo están directamente relacionados con más enfermedad, más medicamentos $\mathrm{y}$ peor pronóstico $\mathrm{y}$, por tanto, podemos asumir que la ausencia de estos datos son una limitación del estudio. Esta circunstancia se tendrá en cuenta en posteriores investigaciones.

Importancia de estos estudios en el panorama farmacéutico actual

En España los datos de notificaciones de RAM de los últimos 33 me- ses en donde interviene el AAS están muy por debajo de la media europea (9/mes en España frente a los 71/mes por país en Europa) $(6,7)$. Una de las razones por las que esto ocurre puede ser sin duda el hecho de que en España la situación de comercialización de los medicamentos con AAS es más restrictiva que en otros países europeos, ya que solo se puede obtener bajo prescripción médica o bien en una farmacia con supervisión del farmacéutico, mientras que en otros países como Reino Unido se puede comprar en un supermercado (9).

El hecho de que en algo más del 5\% de los casos de demanda de un MSP con AAS se haya requerido la intervención del farmacéutico para evitar una RAM, en algunos casos de bastante gravedad, nos hace reflexionar sobre si es apropiado el plantear que el AAS salga de la farmacia comunitaria y se pueda adquirir en otros lugares al permitirse la automedicación sin restricciones.

Este es el primero de un grupo de estudios programados por nuestro grupo que tiene como objetivo el estudio de la importancia de la dispensación por parte del farmacéutico de medicamentos que no precisan prescripción médica, principalmente para evitar RAM que pueden llegar a tener un efecto grave en los pacientes si no se detectan a tiempo

\section{Agradecimientos}

Agradecemos su trabajo a nuestros compañeros farmacéuticos de las farmacias comunitarias que han participado haciendo una realidad este estudio.: Luis Salar Ibáñez, Judith Roa, José Luis Jiménez Carrasco, $\mathrm{M}^{\mathrm{a}}$ Rosario Hernández Marrero, Isabel Vernia Sabater, $M^{\text {a }}$ Pilar Francia, Montserrat Vernia Sabater, Josefina Velert, Andrés Navarro, Vicente J. Baixauli, Pedro del Río Pérez, Garbiñe Olano, Mercedes Bernal Ruiz Mateos, Cristina Márquez Soto, Isabel Benayas Yepes, Cristina Pilar Calvo Francia, María Ángeles Macías Amador, Oliva Pérez Sánchez, José Juan Honrubia y Lourdes Mena. Además queremos agradecer el apoyo en cuestiones informáticas a Ángel Villés (Bitfarma), a Marcelino Valera y Rafael Villalba (Pulso) y a Marc Orcau (Intranet de SEFAC). También agradecer a Jorge Vázquez su apoyo al proyecto y a la empresa HMR la cesión de los datos 
de consumo de medicamentos a nivel nacional. Por último dar las gracias a Francisco Martínez Romero al haber iniciado el proyecto y coordinado su puesta en marcha.

\section{Referencias bibliográficas}

1. Ley de garantías y uso racional de los medicamentos y productos sanitarios. Ley 29/2006, de 26 de julio. Boletín Oficial del Estado nº 178 (27/07/2006). Versión vigente del 26/07/2013.

2. Foro de Atención Farmacéutica. Documento de Consenso. Panorama Actual del Medicamento 2014; 38(375): 645-676.

3. Real Decreto por el que se regula el procedimiento de autorización, registro y condiciones de dispensación de los medicamentos de uso humano fabricados industrialmente. Real Decreto 1345/2007 de 11 de octubre. Boletín oficial del Estado n ${ }^{\circ} 267$ (711-2007).

4. Anónimo. Información sobre medicamentos susceptibles de publicidad en Bot Plus 2.0. [Internet]. Farmacéuticos 2013; 390:36-37. [Consultado el 28 de octubre de 2014] Disponible en: http://publicaciones.portalfarma. com/farmaceuticos/390/files/assets/ basic-html/page36.html

5. Agencia Española de Medicamentos y Productos Sanitarios. Información para las notificaciones de sospechas de reacciones adversas a medicamentos por parte de profesionales sanitarios. 2013. [Internet]. [Consultado el 28 de octubre de 2014]. Disponible en: http://www.aemps.gob.es/vigilancia/medicamentosUsoHumano/ SEFV-H/NRA-SEFV-H/notificaSospechas-RAM-profSanitarios.htm

6. Base de datos europea de informes de presuntas reacciones adversas [Internet]. [Consultado el 30 de octubre de 2014]. Disponible en: http://www. adrreports.eu/es/index.html

7. Sistema Español de Farmacovigilancia de Medicamentos de Usos Humano. FEDRA (Farmacovigilancia Española, Datos de Reacciones Adversas) [Internet]. [Consultado el 30 de octu- bre de 2014]. Disponible en: https:// sinaem.agemed.es/?FEDHUM

8. Williams KA, Emmerton LM, Taylor R, Werner J, Benrimoj SI. Non-prescription medicines and Australian community pharmacy interventions: Rates and clinical significance. Int J Pharm Pract. 2011;19:156-65. doi:10.1111/j.2042-7174.2010.00091.x

9. Consejo General de Colegios Oficiales de Farmacéuticos. Informe: "Los modelos de Farmacia en la Unión Europea” 2007. [Internet]. [Consultado el 30 de octubre de 2014]. Disponible en: http://www.portalfarma.com/Profesionales/farmaciainternacional/Documents/FarmaEuropa2007.pdf

10. Alonso ML, Eyaralar MT, Pérez MA, Jiménez JL, Olano G, Vernia M, et al. Estudio comparativo piloto de la metodología utilizada en los servicios profesionales de las farmacias comunitarias [Internet]. Farmacéuticos Comunitarios. 2014; 6(Supl1). [Consultado el 30 de octubre de 2014]. Disponible en: http://www.farmaceuticoscomunitarios.org/journal-article/ estudio-comparativo-piloto-metodologia-utilizada-servicios-profesionales-farmacias.

11. Abayaratne D, Kurukulaaratchy RJ. Recognising the risk of aspirin-sensitive respiratory disease in a patient with asthma who has previously tolerated aspirin. Prim Care Respir J. 2011;20:214-7. doi:10.4104/ pcrj.2011.00001

12. Babu KS, Salvi SS. Aspirin and asthma. Chest. 2000;118(5):1470-6. doi:10.1378/chest.118.5.1470

13. Awa K, Satoh H, Hori S, Sawada Y. Prediction of time-dependent interaction of aspirin with ibuprofen using a pharmacokinetic/pharmacodynamic model. J Clin Pharm Ther. 2012;37:469-74. doi:10.1111/j.13652710.2011.01313. $\mathrm{x}$

14. Gladding PA, Webster MWI, Farrell HB, Zeng ISL, Park R, Ruijne N. The Antiplatelet Effect of Six Non-Steroidal Anti-Inflammatory Drugs and Their Pharmacodynamic Interaction With Aspirin in Healthy Volunteers. Am J Cardiol. 2008;101:1060-3. doi:10.1016/j.amjcard.2007.11.054
15. Loboz KK, Shenfield GM. Drug combinations and impaired renal function - The "triple whammy."Br J Clin Pharmacol. 2005;59:239-43. doi:10.1111/ j.0306-5251.2004.2188.x

16. Gareri P, Russo G, Merante A, Lacava R, Castagna A, Condito AM, et al. Triple whammy: An obscure threat for elderly patients [Internet]. Ital J Med. 2012;6:64. [Consultado el 30 de octubre de 2014]. doi:10.1016/j. itjm.2012.04.004

17. Kennedy D. Analgesics and pain relief in pregnancy and breastfeeding. Aust Prescr. 2011;34:8-10.

18. Jones L, Othman M, Dowswell T, Alfirevic Z, Gates S, Newburn M, et al. Pain management for women in labour: an overview of systematic reviews. Cochrane database Syst Rev. 2012;3:CD009234.

19. Schrör K. Aspirin and Reye syndrome: A review of the evidence. Pediatr Drugs. 2007;9:195-204. doi:10.2165/00148581200709030-00008

20. Fazakas J, Mándli T, Ther G, Arkossy M, Pap S, Füle B, et al. Evaluation of liver function for hepatic resection. Transplant Proc. 2006;38:798-800. doi:10.1016/j.transproceed.2006.01. 048

21. Doutremepuich C, Aguejouf 0, Desplat V, Eizayaga FX. Paradoxical Effect of Aspirin. Thrombosis. 2012;2012:1-4.

22. De Berardis G, Lucisano G, D’Ettorre A, Pellegrini F, Lepore V, Tognoni $\mathrm{G}$, et al. Association of aspirin use with major bleeding in patients with and without diabetes. JAMA. 20 12;307:2286-94. doi:10.1001/ jama.2012.5034

23. Wong KS, Mok V, Lam WW, Kay R, Tang A, Chan YL, et al. Aspirin-associated intracerebral hemorrhage: clinical and radiologic features. Neurology. 2000;54:2298-301. doi:10.1212/ WNL.54.12.2298

24. Saloheimo P, Ahonen M, Juvela S, Pyhtinen J, Savolainen ER, Hillbom M. Regular aspirin-use preceding the onset of primary intracerebral haemorrhage is an independent predictor for death. Stroke. 2006;37:129-33. doi:10.1161/01. STR.0000196991.03618.31 